\title{
Supporting Information: Functional Fluorescence Microscopy Imag- ing. Quantitative Scanning-Free Confocal Fluorescence Microscopy for the Characterization of Fast Dynamic Processes in Live Cells
}

\author{
Aleksandar J. Krmpot ${ }^{1,2}$, Stanko N. Nikolic ${ }^{1,2}$, Sho Oasa ${ }^{1}$, Dimitrios K. Papadopoulos ${ }^{3, \dagger}$, Marco Vitali ${ }^{4}$, \\ Makoto Oura $^{5}$, Shintaro Mikuni ${ }^{5}$, Per Thyberg ${ }^{6}$, Simone Tisa ${ }^{7}$, Masataka Kinjo ${ }^{5}$, Lennart Nilsson ${ }^{8}$, Lars \\ Terenius $^{1}$, Rudolf Rigler ${ }^{1,9, *}$, Vladana Vukojević ${ }^{1, *}$ \\ ${ }^{1}$ Department of Clinical Neuroscience (CNS), Center for Molecular Medicine (CMM), Karolinska Insti- \\ tutet, 17176 Stockholm, Sweden; ${ }^{2}$ Institute of Physics Belgrade, University of Belgrade, 11080 Bel- \\ grade, Serbia; ${ }^{3}$ Max-Planck Institute for Molecular Cell Biology and Genetics, 01307 Dresden, Germa- \\ ny; ${ }^{4}$ Sicoya GmbH, 12489 Berlin, Germany; ${ }^{5}$ Laboratory of Molecular Cell Dynamics, Faculty of Ad- \\ vanced Life Science, Hokkaido University, Sapporo, 001-0021, Japan; ${ }^{6}$ AlbaNova University Center, \\ Royal Institute of Technology, Department of Applied Physics, 10691 Stockholm, Sweden; ${ }^{7}$ Micro Pho- \\ ton Devices (MPD), 39100 Bolzano, Italy; ${ }^{8}$ Department of Biosciences and Nutrition, Karolinska Insti- \\ tutet, 14183 Huddinge, Sweden; ${ }^{9}$ Department of Medical Biochemistry and Biophysics (MBB), Ka- \\ rolinska Institutet, 17177 Stockholm, Sweden
}

SUMMARY: Description of the software for data analysis, calculation of auto- and cross-correlation curves and image rendering (S1); standard solutions, auxiliary slides for instrument calibration, alignment, and cell culture (S2); optical alignment of the fFMI system (S3); observation volume element size determination (S4); accuracy, precision and single-molecule detection sensitivity of the fFMI system(S5) are given in the Supporting Information (S1-S5) together with the results obtained by imaging in thick specimen the transcription factor Sex Combs Reduced (Scr) in live salivary glands of Drosophila ex vivo (S6).

\section{S1. SOFTWARE FOR DATA ANALYSIS}

Auto-correlation and cross-correlation analysis. Raw data collected by the SPAD camera, consisting of 131000 frames acquired every $20.74 \mu \mathrm{s}$ that yield 1024 fluorescence intensity fluctuation traces recorded over $2.7 \mathrm{~s}$, were stored in the camera's internal memory, transferred to the computer and subjected to correlation analysis to yield auto- and first- and second-order cross-correlation curves (ACC and $\mathrm{CCC}$, respectively) for all $32 \times 32$ pixels in an image frame. For this purpose, the so-called multi-tau algorithm was used, described in detail elsewhere ${ }^{1,2}$. Briefly, in the multi-tau algorithm values of the second order correlation function $G^{(2)}(\tau)$ are determined on a quasi-logarithmic time scale. Each lag time $(\tau)$ for which the $G^{(2)}(\tau)$ value is calculated, is called a channel. The channel is thus characterized by an individual sampling time (the bin width) and the lag time $\tau$ (the delay from the measurement at time 0). The first sixteen channels form the first group, while all other groups consist of eight consecutive channels. The bin width for the first group is determined by the shortest counting interval of the detector, which is 20.74 $\mu \mathrm{s}$ for the SPAD camera used. The following group has an individual sampling time that is twice as long, while for the other channels it is equal to the accumulated sampling time of all preceding channels plus the bin width of its group. Two additional variables are introduced: the so-called delayed monitor $\mathrm{M}_{\text {del }}$ defined for each channel and the direct monitor $\mathrm{M}_{\mathrm{dir}}$ defined for each group. The purpose of $\mathrm{M}_{\mathrm{del}}$ is to accumulate all counts sampled in its channel, while $\mathrm{M}_{\text {dir }}$ accumulates all counts without delay time at a particular sampling time.

The ACCs, $M_{\text {del }}$ and $M_{\text {dir }}$ are calculated according to the following formulas:

$$
\begin{aligned}
& G^{(2)}\left(\tau_{i}\right)=\frac{\frac{1}{M-m} \sum_{k=1}^{M-m} n\left(k \Delta \tau_{i}\right) \cdot n\left(k \Delta \tau_{i}+m \Delta \tau_{i}\right)}{M_{d e l} \cdot M_{d i r}}, \\
& M_{d e l}=\frac{1}{M-m} \sum_{k=m}^{M} n\left(k \Delta \tau_{i}\right) \\
& M_{d i r}=\frac{1}{M-m} \sum_{k=1}^{M-m} n\left(k \Delta \tau_{i}\right)
\end{aligned}
$$

Here, $\tau_{i}$ is the lag time and $\Delta \tau_{i}=2^{i-1} \Delta \tau_{1}$ is the sampling time for channel $i$. Bin width for the first group is $\Delta \tau_{1}=20.74 \mu \mathrm{s}$. 
$m$ and $M$ are integers defined as $m=\tau_{i} / \Delta \tau_{i}$ and $M=T / \Delta \tau_{i}$, where $T$ is the total measurement time. The number of photons counted over a time interval $\left[(k-1) \Delta \tau_{i}, k \Delta \tau_{i}\right]$ is denoted as $n\left(k \Delta \tau_{i}\right)$. In essence, the correlation analysis boils down to obtaining the sum of the products $n\left(k \Delta \tau_{i}\right) \cdot n\left(k \Delta \tau_{i}+m \Delta \tau_{i}\right)$ of the counted photons at time $k \Delta \tau_{i}$ and $m \Delta \tau_{i}$ later, as described by Wohland et al. ${ }^{3}$.

The CCCs are calculated for two SPADs of the camera designated as the "first" or "second" order neighbors of the reference pixel. For example, if the row and column of pixels $a$ and $b$ are denoted as $\operatorname{row}(a), \operatorname{col}(a), \operatorname{row}(b)$ and $\operatorname{col}(b)$, respectively, then pixels $a$ and $b$ are said to be "first" order neighbors if relationships (1) or (2) apply: (1) $|\operatorname{row}(a)-\operatorname{row}(b)|=1 \quad$ and $\quad|\operatorname{col}(a)-\operatorname{col}(b)| \leq 1$ or $\quad$ (2) $|\operatorname{col}(a)-\operatorname{col}(b)|=1$ and $|\operatorname{row}(a)-\operatorname{row}(b)|=0$. Similarly, for "second" order neighbors: (1) $|\operatorname{row}(a)-\operatorname{row}(b)|=2$ and $|\operatorname{col}(a)-\operatorname{col}(b)| \leq 2 \quad$ or $\quad$ (2) $\quad|\operatorname{col}(a)-\operatorname{col}(b)|=2 \quad$ and The following formula was used for the calculation of CCCs:

$$
G_{a b}^{(2)}\left(\tau_{i}\right)=\frac{\frac{1}{M-m} \sum_{k=1}^{M-m} n_{a}\left(k \Delta \tau_{i}\right) \cdot n_{b}\left(k \Delta \tau_{i}+m \Delta \tau_{i}\right)}{M_{a, \text { dir }} M_{b, d e l}},
$$

where $n_{a}\left(k \Delta \tau_{i}\right)$ and $n_{b}\left(k \Delta \tau_{i}\right)$ denote photon counts at time $k \Delta \tau_{i}$ for pixels $a$ and $b$, respectively. $M_{a, \text { del }}$ and $M_{a, d i r}$ are calculated according to (2) and (3) by taking the photon counts $n_{a}$ of pixel $a$. In analogy, $M_{b, d e l}$ and $M_{b, d i r}$ were calculated by taking the photon counts $n_{b}$ of pixel $b$. It is important to note that, in general, $G_{a b}^{(2)}\left(\tau_{i}\right) \neq G_{b a}^{(2)}\left(\tau_{i}\right)$, since the symmetry relation $G_{a b}^{(2)}\left(\tau_{i}\right)=G_{b a}^{(2)}\left(\tau_{i}\right)$ only holds in the absence of directed motion. Thus, for each pair of pixels there are two cross-correlation curves, which may be different in case of directed molecular movement i.e. flow ${ }^{4}$.

Massively parallel calculations of ACC and CCC using the graphics processing unit (GPU). Since fluorescence intensity fluctuations are independently recorded by SPADs that constitute the SPC ${ }^{2}$ camera, parallel computing could be used to speed up data analysis by auto- and cross-correlation. For this purpose, the NVIDIA GeForce GTX 780 graphic card was used that contains 2304 Compute Unified Device Architecture (CUDA) cores that can run tens of thousands of independent tasks (threads) simultaneously. By running as many threads in parallel as possible, the CUDA platform enabled us to use the processing power of the GPU to massively parallelize data analysis. Using one thread to calculate the $G^{(2)}(\tau)$ value of one channel for one particular pixel, the CUDA program executed two groups of threads for ACCs calculation and forty groups of threads for CCCs calculation, where each group runs 64000 threads in parallel on the GPU. This decreased the time required for computation of 1024 ACCs by a central processing unit (CPU) from $\approx 210 \mathrm{~s}$ to $\approx 4 \mathrm{~s}$ by a GPU, and from $\approx 77$ minutes $(\mathrm{CPU})$ to $\approx 45 \mathrm{~s}$ (GPU) for the calculation of CCCs.

fFMI image rendering. An ACC was calculated for each pixel. The amplitude of the ACC was estimated from the value of $\mathrm{G}(\tau)$ at $\tau=103.7 \mu \mathrm{s}$, chosen because the contribution of afterpulsing, which decays quickly, was determined to be negligible for lag times longer than $100 \mu \mathrm{s}$. The average number of molecules in the observation volume element (OVE) was calculated as $N=1 /(\mathrm{G}(103.7)-1))$, and then corrected, $N_{\text {corr }}=$ $N / f_{\text {corr }}$, where the correction factor $f_{\text {corr }}=48.1$ was determined from calibration experiments described in subsection 3.4. The characteristic decay time of the ACC, which is equal to the average translational diffusion time $\left(\tau_{\mathrm{D}}\right)$, was determined from its full width at half maximum. If not otherwise indicated, these values are plotted in fFMI images to show the spatial distribution of molecular numbers $\left(N_{\text {corr }}\right)$ and translational diffusion times $\left(\tau_{\mathrm{D}}\right)$.

For measurements by conventional, spFCS, the experimental ACCs were fitted using the analytical function for free threedimensional (3D) diffusion of a single chemical species and, where appropriate (e.g. Rh6G), triplet formation:

$$
\begin{gathered}
G(\tau)=1+\frac{G_{0}}{\left(1+\frac{\tau}{\tau_{D}}\right) \cdot \sqrt{1+\frac{\omega_{x y}^{2}}{\omega_{z}^{2}} \frac{\tau}{\tau_{D}}}} \\
\cdot\left(1+\frac{T}{1-T} \exp \left(-\frac{\tau}{\tau_{T}}\right)\right)= \\
=1+\frac{1}{N} \cdot \frac{1}{\left(1+\frac{\tau}{\tau_{D}}\right) \cdot \sqrt{1+\frac{\omega_{x y}^{2}}{\omega_{z}^{2}} \frac{\tau}{\tau_{D}}}} \cdot\left(1+\frac{T}{1-T} \exp \left(-\frac{\tau}{\tau_{T}}\right)\right) .
\end{gathered}
$$

In eq. (5), $G_{0}$ is the zero lag time amplitude of the ACC; $N$ is the average number of molecules in the OVE; $\tau_{D}$ is the average diffusion time; $\omega_{x y}$ and $\omega_{z}$ are the radial and the axial radius of the OVE, respectively, where the excitation intensity reaches $1 / e^{2}$ of its value at the center; $T$ is the average equilibrium fraction of molecules in the triplet state (when not applicable, $T=0$ ); and $\tau_{T}$ is the triplet correlation time, related to the rate constants for intersystem crossing and the triplet decay. The diffusion coefficient $D$ was determined from the diffusion time using the relationship $\tau_{D}=\omega_{x y}^{2} / 4 D$.

\section{S2. STANDARD SOLUTIONS, AUXILIARY SLIDES FOR INSTRUMENT CALIBRATION, ALIGNMENT, AND CELL CULTURE.}

Laser grade Rhodamine 6G (Rh6G), dye content $99 \%$ (Sigma-Aldrich), $D_{\text {Rh6G }}=4.14 \times 10^{-10} \mathrm{~m}^{2} \mathrm{~s}^{-1}$, was used to prepare standard solutions for spFCS instrument calibration. Dilute aqueous suspension of yellow-green fluorescent carboxylatemodified polystyrene nano/microspheres of different diameter: $d=20 \mathrm{~nm}, 100 \mathrm{~nm}\left(\mathrm{D}_{\mathrm{fs} 100}=4.4 \times 10^{-12} \mathrm{~m}^{2} \mathrm{~s}^{-1},\right), 200 \mathrm{~nm}, 500$ $\mathrm{nm}, 1.0 \mu \mathrm{m}$ and $2.0 \mu \mathrm{m}$ (FluoSpheres ${ }^{\circledR}$ Size Kit \#2; Ex/Em: $505 / 515)$ and carboxylate functionalized quantum dot nano- 
crystals, $d=20 \mathrm{~nm}$, emission maxima at $525 \mathrm{~nm}$ (Qdot@ 525 ITK ${ }^{\text {TM }}$ Carboxyl Quantum Dots), Molecular Probes, Life Technologies Corporation, USA, were used for fFMI instrument calibration and performance characterization. For this purpose quantum dots/fluospheres suspension were freshly prepared and sonicated in an ultrasonic bath for $30 \mathrm{~min}$ before use.

For fFMI instrument alignment, a uniform thin layer of Rh6G was prepared by squeezing $1 \mu \mathrm{L}$ of concentrated Rh6G solution in water between a microscopic slide and a cover glass (\#1.5 thickness, $22 \times 40 \mathrm{~mm}$ ) and allowed to dry.

Preparation of fixed muntjac skin fibroblast cells with filamentous actin stained using Alexa Fluor ${ }^{\circledR} 488$ phalloidin (FluoCells ${ }^{\circledR}$ Prepared Slide \#6; Muntjac cells with Mouse Anti-OxPhos Complex V Inhibitor Protein, Alexa Fluor ${ }^{\circledR} 555$ Goat Anti-Mouse IgG, Alexa Fluor ${ }^{\circledR} 488$ Phalloidin, and TOPRO ${ }^{\circledR}-3$ ) was purchased from Molecular Probes, Life Technologies Corporation, USA and used for fFMI performance characterization.

Live salivary glands ex vivo. Third instar wandering Drosophila larvae expressing dimers of the Hox transcription factor Sex combs reduced (Scr) coupled to the mCitrine fluorescent protein and a multimer Scr-binding site of the fork-head enhancer $\left(f k h 250^{\text {con }}\right)$, as previously described ${ }^{5-7}$, were dissected in phosphate buffered saline ( $\mathrm{PBS}, \mathrm{pH}=7.4$ ) at room temperature. The salivary glands were transferred to 8 -well chambered cover glass (Nunc ${ }^{\circledR}$ Lab-Tek ${ }^{\circledR}$ II, Thermo Fisher Scientific, USA) containing $200 \mu \mathrm{L}$ of PBS for imaging.

Cell culture. PC12 and U-2 OS cells were purchased from the American Type Culture Collection (ATCC). PC12 cells were stably transformed to express the $\mu$-opioid receptor genetically fused with the enhanced Green Fluorescent Protein at the $\mathrm{C}$ terminus (eGFP-MOP) $)^{8,9}$. U-2 OS cells were transiently transformed to express the eGFP monomer (eGFP), eGFP tetramer $\left(\mathrm{eGFP}_{4}\right)$ consisting of four eGFP molecules covalently bound via a flexible linker, or the glucocorticoid receptor tagged with eGFP at the $\mathrm{N}$ terminus $\left(\mathrm{eGFP}-\mathrm{GR}_{\mathrm{wt}}\right)^{10}$.

For multiplication purposes PC12 cells were cultured in collagen-coated flasks using RPMI 1640 medium supplemented with $5 \%$ fetal bovine serum (FBS), $10 \%$ heat-inactivated horse serum, $100 \mathrm{U} / \mathrm{ml}$ penicillin and $100 \mu \mathrm{g} / \mathrm{ml}$ streptomycin; whereas U-2 OS cells were grown in the Dulbecco's modified Eagle's medium (DMEM) supplemented with $10 \%$ FBS. All cell culture reagents were from Invitrogen, Sweden. The cells were maintained at $37{ }^{\circ} \mathrm{C}$ in a humidified $5 \% \mathrm{CO}_{2}$ atmosphere and sub-cultured every 3-4 days. For FCS experiments, PC12 cells were plated in 8-well chambered cover glass (Nunc ${ }^{\circledR}$ Lab-Tek ${ }^{\circledR}$ II, Thermo Fisher Scientific, USA) 2-3 days before the FCS experiment and grown in phenol-red free RPMI cell culture medium.

The U-2 OS cells were plated in the 8-well chambered cover glass at a cell density of $\sim 10,000$ cells per well one day before transfection. The cells were transfected with $300 \mathrm{ng}$ plasmid DNAs for transient expression of eGFP-GR ${ }_{w}$, eGFP monomer or $\mathrm{eGFP}_{4}$ tetramer using $2.6 \mu \mathrm{L}$ Lipofectamine 2000. Three hours after transfection, the cell culture medium was replaced by a fresh medium supplemented with $10 \%$ FBS. The trans- fected U-2 OS cells were then cultured for 21 hours and subjected to FCS measurements. All FCS experiments were performed at room temperature.

In order to induce eGFP-GR $\mathrm{wt}_{\mathrm{wt}}$ nuclear translocation, U-2 OS cells were treated with $100 \mathrm{nM}$ Dexamethasone (Dex), purchased from Sigma (St.Louis, MO, USA).

\section{S3. OPTICAL ALIGNMENT OF THE fFMI SYSTEM}

To align the illumination and detection matrices, the dried Rh6G sample was illuminated and the spot-wise image was recorded using the DSLR camera. The pitch of the illumination matrix was adjusted by translating the DOE along the beam axis (Fig. 1 a). Thereafter, light was directed to the SPAD camera attached to the other side port of the microscope. Photon counts were observed in all SPADs in real time using a software routine specially designed for this purpose. By moving the focusing lens (Fig. 1 a) in the plane perpendicular to the laser beam the lateral position of the illumination matrix was adjusted until the signal was maximized in all SPADs simultaneously, indicating that the system is well aligned (Fig. S1 a).
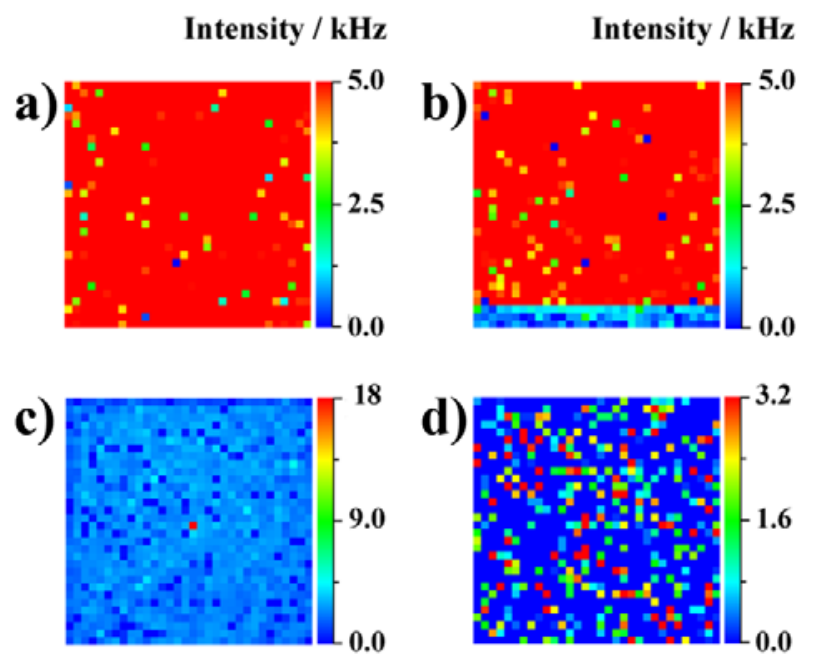

Figure S1. Optical alignment of the fFMI system. a) Image of dried Rh6G obtained using the SPAD matrix detector when the fFMI system is well aligned. b) Image of dried Rh6G obtained using a deliberately misaligned fFMI system, where the illumination and detection matrices are shifted three rows between one another. c) Image of dried Rh6G acquired using a deliberately misaligned fFMI system, where individual illumination spots fall between individual SPADs and the $0^{\text {th }}$-order maximum, which in a well aligned system is not visible as it falls between the central pixels, is captured in the red pixel $(17,16)$. d) Dark count rate distribution in the SPAD camera.

In order to verify that the illumination and detection matrices overlap, we show here two examples of deliberate misalignment: (1) when there is a mismatch of three rows between the illumination and detection matrices (Fig. S1 b); and (2) when the zero-order diffraction spot, which normally is not visible as it falls between the central SPADs on the SPAD matrix detector, is deliberately visualized in pixel $(17,16)$ (Fig. S1 c, red pixel). Since all illumination spots in the latter case fall 
between the SPADs, the signal recorded in all other SPADs is very low (Fig. S1 c, blue) and the measured intensity is at the level of the detector dark count (Fig. S1 d).

\section{S4. OBSERVATION VOLUME ELEMENT SIZE}

To determine the OVE size, a suspension of fluospheres, $d=$ $100 \mathrm{~nm}$, with a known diffusion coefficient $\left(\mathrm{D}=4.4 \times 10^{-12}\right.$ $\left.\mathrm{m}^{2} \mathrm{~s}^{-1}\right)^{11}$, was used as a calibration standard. The spatial distribution of diffusion times was mapped (Fig. S2 a), and the corresponding histogram of diffusion time distribution was obtained (Fig. S2, b), yielding an average diffusion time $\tau_{D}=$ $(3.7 \pm 1.1) \mathrm{ms}$

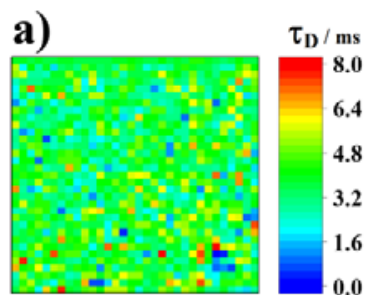

c)

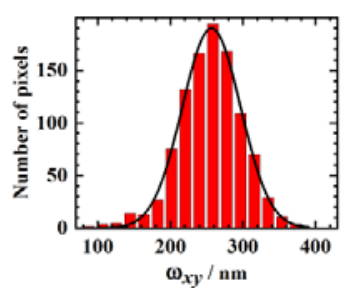

e)

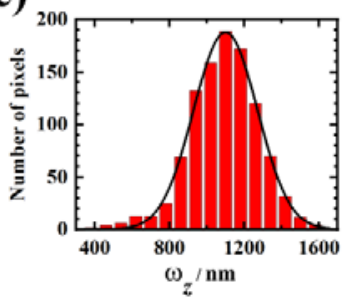

b)

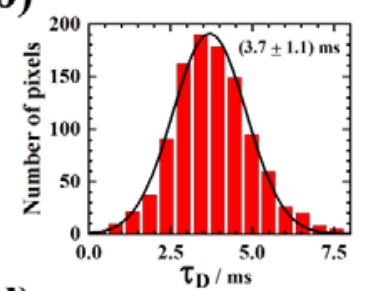

d)

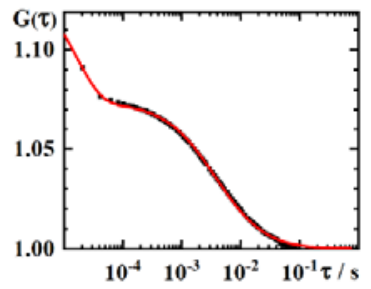

f)

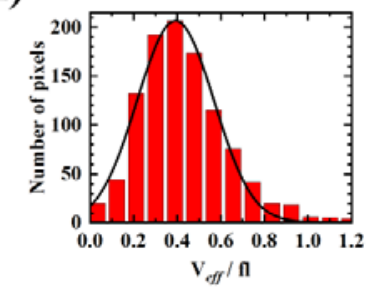

Figure S2. Observation volume element size. a) Diffusion time map recorded in an aqueous suspension of fluospheres, $d$ $=100 \mathrm{~nm}$. b) Corresponding histogram of diffusion times distribution. The average diffusion time was determined to be $\tau_{\mathrm{D}}$ $=(3.7 \pm 1.1) \mathrm{ms}$. c) Histogram of lateral radius size distribution, $\omega_{x y}=(255 \pm 40) \mathrm{nm}$. d) Average ACC across all pixels (black squares) and the fitted ACC (red). e) Histogram of axial radius size distribution, $\omega_{z}=(1.1 \pm 0.2) \mu \mathrm{m}$. f) Histogram of effective volume size distribution, $\mathrm{V}_{\text {eff }}=(0.36 \pm 0.17) \times 10^{-15} 1$.

The lateral radius size was then calculated for each OVE using the relationship $\tau_{D}=\omega_{x y}^{2} / 4 D$, i.e. $\omega_{x y}^{2}=4 \cdot D^{\cdot} \tau_{D}$, yielding a map of lateral radii size (data not shown) and the average size of the lateral radius was determined from the histogram of lateral radius size distribution, $\omega_{x y}=(255 \pm 40) \mathrm{nm}$ (Fig. S2 c).

To determine the axial radius, an average ACC across the SPAD matrix was generated (Fig. S2 d, black squares) and fitted using a theoretical equation that is analogous to equation (5), where detector afterpulsing visible at lag times $\tau_{D}<40 \mu \mathrm{s}$ was represented by an exponential decay term (Fig. S2 d, red curve $)$. Fitting yielded the diffusion time, $\tau_{D}=(3.7 \pm 1.1) \mathrm{ms}$ and the axial to lateral structure parameter ratio, $\omega_{z} / \omega_{x y}=$ 4.28. Using this value and the average lateral radius size, the axial radius size was determined to be $\omega_{z}=(1.1 \pm 0.2) \mu \mathrm{m}$ (Fig. S2 e).

Finally, the effective volume was calculated $\mathrm{V}_{\text {eff }}=$ $\pi^{3 / 2} \cdot \omega_{x y}^{2} \cdot \omega_{z}$ for each pixel (data not shown), and the average effective volume of the OVE was determined from the histogram of effective volume size distribution, $V_{\text {eff }}=(0.36 \pm$ $0.17) \times 10^{-15} 1$ (Fig. S2 f).

\section{S5. ACCURACY, PRECISION AND SINGLE- MOLECULE DETECTION SENSITIVITY OF THE fFMI SYSTEM}

While diffusion times can be determined with great precision and the diffusion coefficients obtained by fFMI agreed well with values determined by spFCS and with theoretically calculated values (Fig. S3 a), the distribution of diffusion times measured in the suspension of fluospheres is somewhat broad (Fig. 1 e) for several reasons. Most notably, the fluospheres suspension contains, despite prolonged sonication, not only individual fluospheres but also some agglomerates, which are now more readily detected since we examine 1024 OVEs simultaneously. Furthermore, the intensity of the incident light in individual foci is rather weak, estimated to be $1 / 1024$ of the intensity measured at the microscope objective $(18.9 \mathrm{~mW})$ reduced by $20 \%$ to account for the intensity of the zero-order diffraction peak. Consequently, the autocorrelation curves are somewhat noisier. Finally, the diffusion time is determined by reading out the full width at half-maximum from the experimentally derived ACCs, which are noisy, rather than from the fitted theoretical ACCs.

In comparison to diffusion times, the amplitude of the autocorrelation curve determined by the fFMI and the spFCS differed considerably (Fig. S3 b).

The difference in the amplitudes of ACCs arises primarily due to differences in the signal-to-noise-ratio (SNR) between the fFMI and the spFCS systems. The primary contributor to lower SNR ratio in the fFMI system is the dark count rate of individual SPADs, which for most SPADs comprising the matrix detector was over 1000 counts per second for individual SPAD (Fig. S1 d). and less than 250 photons per second in the conventional spFCS system used as a reference ${ }^{12}$.

Another important contributing factor is the so-called pinhole cross-talk, i.e. increase in the background signal arising due to inadvertent transmission of out-of-focus light through neighboring detectors. Finally, when 1024 ACCs are simultaneously recorded, the signal acquisition time length in the fFMI system is limited to $2.7 \mathrm{~s}$ by the internal memory of the matrix SPAD camera, which may restrain the underlying statistical analysis resulting in noisy ACCs. By improving these features, the concentration and diffusion of eGFP molecules in water could be characterized (Fig. S3 c-f). Here, the amplitude of the average $\mathrm{ACC}$ recorded by the fFMI system, $\mathrm{G}_{0, \mathrm{fFMI}}=$ $1.05 \pm 0.01$ (Fig. S3 d, black), differed from the value measured by 


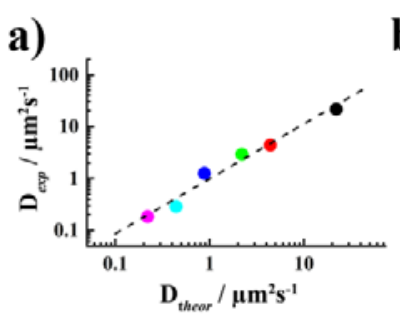

b) $\mathrm{N}$
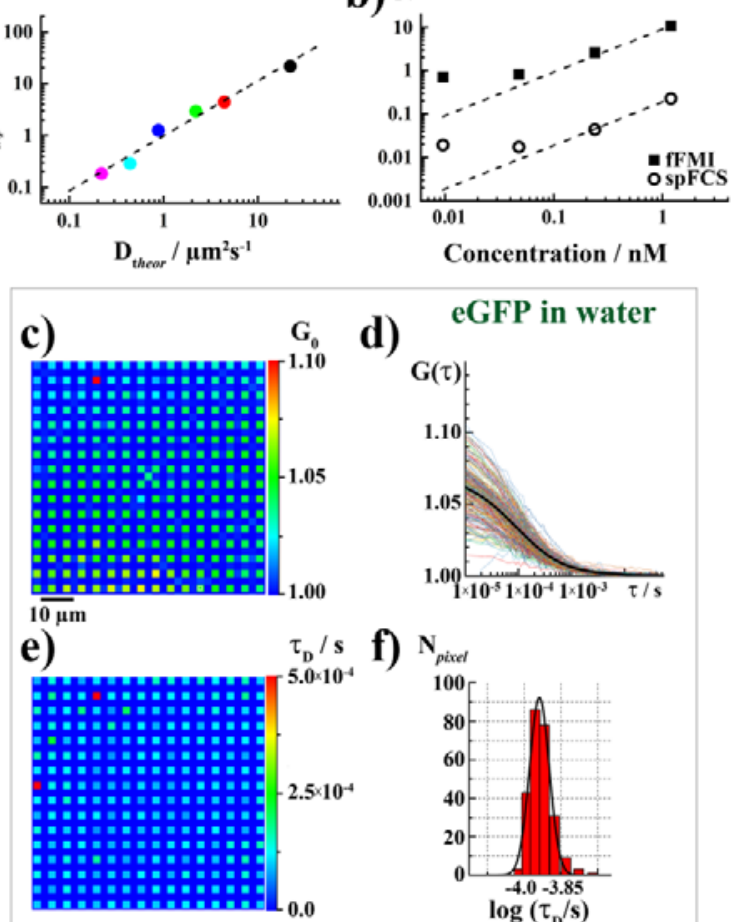

d)

eGFP in water

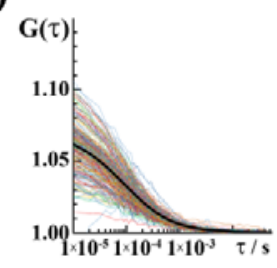

f)

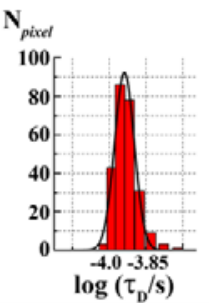

Figure S3. Accuracy, precision and single-molecule detection sensitivity of the ffmi system. a) Diffusion coefficient of fluospheres of different diameter: $d=0.02 \mu \mathrm{m}$ (black), $d=0.1$ $\mu \mathrm{m}$ (red), $d=0.2 \mu \mathrm{m}$ (green), $d=0.5 \mu \mathrm{m}$ (blue), $d=1.0 \mu \mathrm{m}$ (cyan) and $d=2.0 \mu \mathrm{m}$ (pink), experimentally measured by fFMI, with respect to theoretically expected values. b) Amplitudes of ACCs measured in a dilute suspension of fluospheres, $d=100 \mathrm{~nm}$, by fFMI (squares) and spFCS (open circles). For all concentrations tested, the amplitude measured by spFCS was 48.1 times larger than the amplitude by fFMI, as determined from the ratio of slopes of the regression equations: $y=$ $(0.189 \pm 0.005) \cdot x$, for $\mathrm{fFMI}$, and $y=(9.1 \pm 0.4) \cdot x$, for $\mathrm{spFCS}$, yielding the correction factor $f_{\text {corr }}=9.1 / 0.189=48.1$. The fFMI and spFCS measurements were performed at comparable excitation intensities $(14.8 \mu \mathrm{W}$ at the microscope objective lens in spFCS). Background correction was not applied. Box: c) Spatial map of amplitudes $\left(\mathrm{G}_{0}\right)$ acquired by fFMI in a $(18.5$ $\pm 0.4) \mathrm{nM}$ solution of eGFP in water (eGFP concentration was determined by the spFCS system). d) Individual ACCs acquired by fFMI in the same sample described in i). The amplitude $\mathrm{G}_{0}$ of the average ACC (solid black) was $\mathrm{G}_{0, \mathrm{fFMI}}=(1.05 \pm$ $0.01)$. e) Spatial map of translational diffusion times acquired by fFMI in the same sample described in c). f) Corresponding histogram of translation diffusion time distribution. Gaussian fitting yields translational diffusion time for eGFP in water, $\tau_{\text {D.eGFP }}=(110 \pm 30) \mu \mathrm{s}$. In c) $\left.-\mathrm{f}\right)$, the inter-pixel distance was increased using a $16 \times 16 \mathrm{DOE}$ and every other detector on the next generation SPAD camera. Signal acquisition time was four times longer, as the number of simultaneously acquired ACCs was reduced from 1024 to 256 .

$\operatorname{spFCS}, \mathrm{G}_{0, \mathrm{spFCS}}=1.26 \pm 0.01,4.9$ times $\left(f_{\text {corr }}=4.9\right)$. Similarly, the translational diffusion times, $\tau_{\mathrm{D}, \mathrm{fFMI}}=(120 \pm 30) \mu \mathrm{s}$ and $\tau_{\mathrm{D}, \mathrm{spFCS}}=(85 \pm 3) \mu \mathrm{s}$, yield, after correction for differences in

the observation volume area size $\left(\omega_{x y, s p F C S}^{2}=0.042 \mu \mathrm{m}^{2}\right.$ and $\left.\omega_{x y, f F M I}^{2}=0.054 \mu \mathrm{m}^{2}\right)$, diffusion coefficient values that agree within the experimental error $\left(D_{\mathrm{spFCS}}=(1.2 \pm 0.1) \times 10^{-10} \mathrm{~m}^{2} \mathrm{~s}^{-1}\right.$ and $\left.\mathrm{D}_{\mathrm{fFMI}}=(1.1 \pm 0.1) \times 10^{-10} \mathrm{~m}^{2} \mathrm{~s}^{-1}\right)$. Importantly, these values agree also with literature values, $D_{\text {eGFP }}=0.95 \times 10^{-10} \mathrm{~m}^{2} \mathrm{~s}^{-1} 13$.

\section{S6. IMAGING THICK SPECIMEN USING fFMI}

While we have established that crosstalk between pixels is not an issue for quantitative characterization of concentration and diffusion in dilute solutions/suspensions (Fig. 1 f), it is well known that the main challenge for quantitative fluorescence microscopy imaging of thick specimen using multi-focal optical arrangement arises because out-of-focus light that originates from bright structures in remote focal planes above/below the sample plane can pass through adjacent pinholes. This increases the background signal, i.e. reduces the SNR, and gives rise to hazy images where details that are normally observed in confocal laser scanning microscopy are obscured in spinning disk confocal microscopy ${ }^{14}$. In order to probe the capacity of the fFMI system to characterize dynamical processes in thick samples, the concentration and nuclear dynamics of the mCitrine-tagged Sex combs reduced (Scr) dimeric transcription factor (mCitrine-(Scr $)_{2}$ ) was investigated in salivary glands from Drosophila third instar larvae bearing in the genome a multimeric specific binding site of Scr ( $f k h 250^{\text {con }}$; see Materials and Methods and ${ }^{15}$ for details). The obtained results are presented in Fig. S4.

Fluorescence intensity imaging of a polytene nucleus acquired by the DSLR camera is shown in Fig. S4 a. The spatial map of the average number of mCitrine-(Scr $)_{2}$, calculated by extrapolating to zero lag time the virtually noise-free $G(\tau)$ values at lag time $\tau=5 \mathrm{~ms}$, is shown in Fig. S4 b, and the corresponding spatial map of diffusion times in Fig. S1 c. The histogram reflecting the distribution of diffusion times inside the cell nucleus is shown in Fig. S4 d. Fluorescence intensity fluctuations simultaneously recorded at several different positions in the cell nucleus (Fig. S4 e, yellow, blue and magenta) and in the cytoplasm (Fig. S4 e, black), show that the average signal intensity is unchanged during the signal acquisition time $(2.7 \mathrm{~s})$, indicating that the signal is not distorted by extensive photobleaching. Individual ACCs acquired in different pixels in the cell nucleus are shown in Fig. S4 f.

fFMI maps of the average number of mCitrine-(Scr $)_{2}$ molecules (Fig. S4 b) and their translational diffusion time (Fig. $\mathrm{S} 4 \mathrm{c})$, clearly show that the mCitrine-(Scr $)_{2}$ transcription factor is predominantly located in the cell nucleus (Fig. S4 b), and that nuclear diffusion is rather slow (Fig. S4 c and d), presumably due to interactions with the multimeric specific binding site of Scr. Furthermore, fFMI shows that the investigated protein is not uniformly distributed in the cell nucleus, and that domains with different mCitrine-(Scr $)_{2}$ concentration and diffusion exist. This is expected, since polytene cell nuclei contain $2^{10}$ chromosomal copies associated together in giant polytene chromosomes. In the nucleoplasm, where there is no chromatin, the diffusion of unbound mCitrine-(Scr $)_{2}$ is faster because its movement is not deterred by interactions with the DNA $^{5,6}$. 


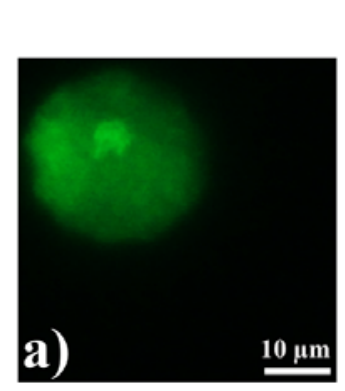

e)

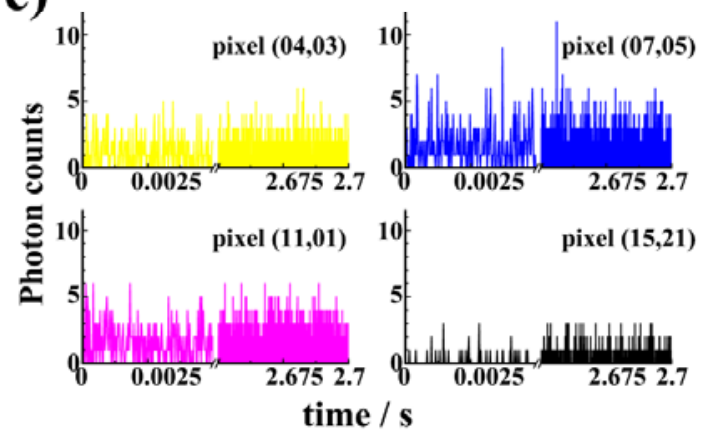

$\mathbf{N}_{\text {corr }}$

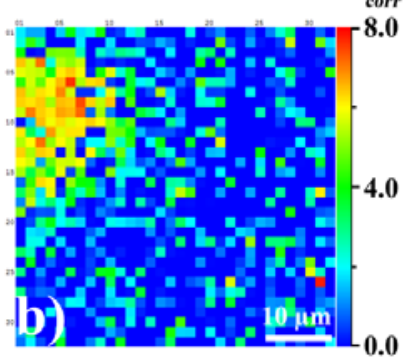

0.0

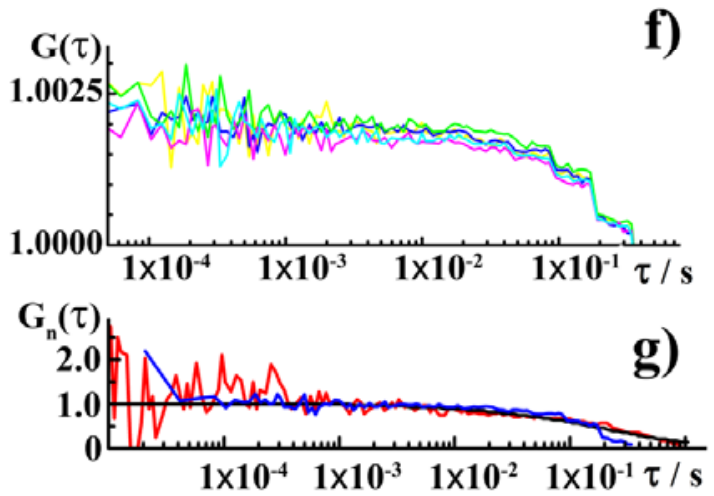

Figure S4. Spatial distribution of molecular numbers and diffusion time maps of transcription factor dimers in live salivary glands of Drosophila. a) DSLR image of a polytene cell nucleus in a live salivary gland dissected from a third instar larva of the fruit fly Drosophila melanogaster genetically engineered to express a mCitrine-tagged dimeric Sex Combs Reduced (Scr) transcription factor mCitrine-(Scr $)_{2}$. Note that only the cell nucleus is unambiguously visible in the images, while the fluorescence intensity in the cytoplasm was at the level of background. b) Spatial map of molecular numbers $\left(\mathrm{N}_{\text {corr }}\right)$ in the nucleus shown in a) (slightly shifted due to differences in the position of the images), shows uneven transcription factor distribution. c) Spatial map of diffusion times $\left(\tau_{\mathrm{D}}\right)$ reveals that transcription factor dynamic behavior is non-uniform, showing domains where fast or slow diffusion prevails. Regions where slow diffusion is observed reflect transcription factor binding to chromatin, i.e. putative sites of transcriptional activity. d) Corresponding diffusion time histogram (pixels in rows 3-16 and columns 1-10). e) Fluorescence intensity fluctuations (photon counts per $20.74 \mu \mathrm{s}$ ) recorded in selected pixels in the cell nucleus (yellow, blue and magenta) and the cytoplasm (black). Time series collected during $2.7 \mathrm{~s}$ show that significant photobleaching was not observed during signal acquisition. f) Corresponding ACCs recorded in the selected pixels in the cell nucleus, generated by temporal autocorrelation analysis of fluorescence intensity fluctuations shown in e). g) ACCs normalized to the same amplitude $(\mathrm{G}(\tau)=1$ at $\tau=103.7 \mu \mathrm{s})$ recorded by fFMI (blue) and conventional FCS instrument used as a reference (red). The black line represents the fitting using an autocorrelation function derived for a model for free three-dimensional diffusion (eq. (5)). The overlap between ACCs shows that optical properties of the newly developed instrument are of high quality and that the observation volume element size is similar in both setups.

On the average, the amplitudes of ACCs acquired by fFMI were estimated to be about 50-60 times smaller than the amplitudes of ACCs acquired spFCS. (As it was not always possible to perform measurements on exactly the same cell using both systems, the difference in amplitudes was estimated at the population level from measurements on 10 cells in the same salivary gland.) This value is somewhat larger than the value determined for in solution measurements, $f_{\text {corr }}=48.1$, suggesting that the SNR is lower. However, the decay times of the ACCs acquired by fFMI (Fig. S4 g, blue) and by conventional spFCS (Fig. S4 g, red), agreed well, as can be seen from the overlap of ACC normalized to the same amplitude (Fig. S4 g), and ACCs acquired by fFMI showed similar value for the translational diffusion time, $\tau_{\mathrm{D}, \mathrm{fFMI}} \approx \tau_{\mathrm{D}, \mathrm{FCS}}=25 \mathrm{~ms}$, as was obtained by fitting the ACCs obtained by spFCS using eq. 5 (Fig. S4 g, black). This is expected for FCS measurements in thick samples. Here, the amplitudes of ACCs change due to the uncorrelated crosstalk from deeper tissue sections, which increases the background and reduces the amplitude of the ACCs. However, the decay time of the ACCs is not influenced, and the diffusion time can be correctly determined. 


\section{REFERENCES}

1. Schatzel, K.; Drewel, M.; Stimac, S., Photon-Correlation Measurements at Large Lag Times - Improving Statistical Accuracy. J Mod Optic 1988, 35 (4), 711-718.

2. Schatzel, K., Accuracy of Photon-Correlation Measurements on Nonergodic Samples. Appl Optics 1993, 32 (21), 38803885 .

3. Wohland, T.; Rigler, R.; Vogel, H., The standard deviation in fluorescence correlation spectroscopy. Biophys $J$ 2001, 80 (6), 2987-2999.

4. Magde, D.; Webb, W. W.; Elson, E. L., Fluorescence Correlation Spectroscopy .3. Uniform Translation and Laminar-Flow. Biopolymers 1978, 17 (2), 361-376.

5. Vukojevic, V.; Papadopoulos, D. K.; Terenius, L.; Gehring, W. J.; Rigler, R., Quantitative study of synthetic Hox transcription factor-DNA interactions in live cells. Proceedings of the National Academy of Sciences of the United States of America 2010, 107 (9), 4093-8.

6. Papadopoulos, D. K.; Vukojevic, V.; Adachi, Y.; Terenius, L.; Rigler, R.; Gehring, W. J., Function and specificity of synthetic Hox transcription factors in vivo. Proceedings of the National Academy of Sciences of the United States of America 2010, 107 (9), 4087-92.

7. Papadopoulos, D. K.; Skouloudaki, K.; Adachi, Y.; Samakovlis, C.; Gehring, W. J., Dimer formation via the homeodomain is required for function and specificity of Sex combs reduced in Drosophila. Developmental biology 2012, 367 (1), 78-89.

8. Rogacki, M. K.; Golfetto, O.; Tobin, S. J.; Li, T.; Biswas, S.; Jorand, R.; Zhang, H.; Radoi, V.; Ming, Y.; Svenningsson, P.; Ganjali, D.; Wakefield, D. L.; Sideris, A.; Small, A. R.; Terenius, L.; Jovanovic-Talisman, T.; Vukojevic, V., Dynamic lateral organization of opioid receptors (kappa, muwt and muN40D ) in the plasma membrane at the nanoscale level. Traffic 2018, 19 (9), 690709 .
9. Tobin, S. J.; Wakefield, D. L.; Terenius, L.; Vukojevic, V.; Jovanovic-Talisman, T., Ethanol and Naltrexone Have Distinct Effects on the Lateral Nano-organization of $\mathrm{Mu}$ and Kappa Opioid Receptors in the Plasma Membrane. ACS chemical neuroscience 2019, 10 (1), 667-676.

10. Mikuni, S.; Pack, C.; Tamura, M.; Kinjo, M., Diffusion analysis of glucocorticoid receptor and antagonist effect in living cell nucleus. Experimental and molecular pathology 2007, 82 (2), 163-8.

11. Kapusta, P. Absolute Diffusion Coefficients: Compilation of Reference Data for FCS Calibration. Application Note [Online], 2010.

12. Vukojevic, V.; Heidkamp, M.; Ming, Y.; Johansson, B.; Terenius, L.; Rigler, R., Quantitative single-molecule imaging by confocal laser scanning microscopy. Proceedings of the National Academy of Sciences of the United States of America 2008, 105 (47), 18176-81.

13. Petrasek, Z.; Schwille, P., Precise measurement of diffusion coefficients using scanning fluorescence correlation spectroscopy. Biophys J 2008, 94 (4), 1437-48.

14. Shimozawa, T.; Yamagata, K.; Kondo, T.; Hayashi, S.; Shitamukai, A.; Konno, D.; Matsuzaki, F.; Takayama, J.; Onami, S.; Nakayama, H.; Kosugi, Y.; Watanabe, T. M.; Fujita, K.; Mimori-Kiyosue, Y., Improving spinning disk confocal microscopy by preventing pinhole cross-talk for intravital imaging. Proceedings of the National Academy of Sciences of the United States of America 2013, 110 (9), 3399404.

15. Papadopoulos, D. K.; Krmpot, A. J.; Nikolic, S. N.; Krautz, R.; Terenius, L.; Tomancak, P.; Rigler, R.; Gehring, W. J.; Vukojevic, V., Probing the kinetic landscape of Hox transcription factor-DNA binding in live cells by massively parallel Fluorescence Correlation Spectroscopy. Mechanisms of development 2015, 138 Pt 2, 218-225. 